\title{
An evaluation of techniques for dose calculation on cone beam computed tomography
}

\author{
'VALENTINA GIACOMETTI, PhD, 1,2 RAYMOND B. KING, PhD, ${ }^{2}$ CHRISTINA E. AGNEW, PhD, ${ }^{2}$ DENISE M. IRVINE, PhD, \\ 1,2 SUNEIL JAIN, MBBCh, PhD, ${ }^{1,2}$ ALAN R. HOUNSELL, PhD and ${ }^{1,2}$ CONOR K. MCGARRY, PhD \\ ${ }^{1}$ Centre for Cancer Research and Cell Biology, Queen's University Belfast, Belfast, UK \\ ${ }^{2}$ Radiotherapy Physics, Northern Ireland Cancer Centre, Belfast, UK \\ Address correspondence to: Dr Valentina Giacometti \\ E-mail:vale.jac@hotmail.it
}

Objective: To assess the accuracy and efficiency of four different techniques, thus determining the optimum method for recalculating dose on cone beam $\mathrm{CT}$ (CBCT) images acquired during radiotherapy treatments.

Methods: Four established techniques were investigated and their accuracy assessed via dose calculations: (1) applying a standard planning CT ( $\mathrm{pCT}$ ) calibration curve, (2) applying a CBCT site-specific calibration curve, (3) performing a density override and (4) using deformable registration. Each technique was applied to 15 patients receiving volumetric modulated arc therapy to one of three treatment sites, head and neck, lung and prostate. Differences between $\mathrm{PCT}$ and $\mathrm{CBCT}$ recalculations were determined with dose volume histogram metrics and 2.0\%/O.1 mm gamma analysis using the PCT dose distribution as a reference.

Results: Dose volume histogram analysis indicated that all techniques yielded differences from expected

\section{INTRODUCTION}

Image-guided radiotherapy (IGRT) has advanced significantly following the incorporation of $\mathrm{kV}$-based on-board imagers (OBIs) with treatment linear accelerators (linacs). ${ }^{1}$ In addition to acquiring high quality two-dimensionally planar radiographs and fluoroscopy images, OBIs are capable of generating volumetric cone beam CT (CBCT) images of a patient's internal anatomy, ${ }^{2}$ enabling more accurate positioning of patients prior to their treatment. ${ }^{3}$ Due to their acquisition arrangement, the three-dimensional (3D) CBCT images generated by OBIs are of inferior quality compared to the planning CTs ( $\mathrm{pCTs}$ ), generated by diagnostic CT scanners used to plan a patient's radiotherapy treatment. ${ }^{4}$ However, there have been a number of studies investigating the accuracy of treatment planning dose calculations performed using CBCT images and potential methods to improve observed limitations in their results between 0.0 and $2.3 \%$ for both target volumes and organs at risk. With volumetric gamma analysis, the dose recalculation on deformed images yielded the highest pass-rates. The median pass-rate ranges at 50\% threshold were 99.6-99.9\%, 94.6-96.0\%, and 94.8.0$96.0 \%$ for prostate, head and neck and lung patients, respectively.

Conclusion: Deformable registration, $\mathrm{HU}$ override and site-specific calibration curves were all identified as dosimetrically accurate and efficient methods for dose calculation on CBCT images.

Advances in knowledge: With the increasing adoption of $\mathrm{CBCT}$, this study provides clinical radiotherapy departments with invaluable information regarding the comparison of dose reconstruction methods, enabling a more accurate representation of a patient's treatment. It can also integrate studies in which $\mathrm{CBCT}$ is used in image-guided radiation therapy and for adaptive radiotherapy planning processes. calculation accuracy. ${ }^{5-11}$ Dose calculations derived from anatomy outlined on CBCT have been used to determine more accurate estimates of the physical dose that patients actually receive during prostate radiotherapy treatments. ${ }^{12}$ This information can allow for improved comparisons with the patient's treatment outcome $\mathrm{e}^{13}$ and can also be employed in adaptive therapy strategies ${ }^{9,14}$ informing clinicians of the dosimetric consequences of significant changes in the patient's anatomy.

One of the main limitations to CBCT images is that a large volume of the patient is simultaneously irradiated during their acquisition. This impedes the reduction of scattered photon contribution to the transmitted projections used to reconstruct the $\mathrm{CBCT}$ images, ${ }^{4,8}$ as well as reducing image quality. By impacting image contrast and increasing image artefacts, the increased scatter component also influences 
the relationship between CT pixel numbers (expressed in Hounsfield units-HU) and the attenuation coefficient of the patient's tissues. ${ }^{9}$ With pCT images this relationship remains relatively constant and the $\mathrm{HU}$ pixel data can be converted to a corresponding electron density $\left(\rho_{\mathrm{e}}\right)$ to model how megavoltage (MV) photons will interact and deposit dose in patient tissue. However, the increased contribution of scattered photons to CBCT projections means that the relationship between $\mathrm{HU}$ and electron density is no longer fixed and may vary depending on patient size and/or the region of anatomy being imaged. ${ }^{9}$

A number of potential techniques for performing treatment planning calculations on CBCT images have been proposed, ${ }^{5-11,15,16}$ with the execution of these techniques ranging in complexity. A number of studies have employed the standard pCT HU to $\rho_{\mathrm{e}}$ calibration curve to perform dose calculations on CBCT images and observed adequate accuracy. ${ }^{5,6,10,12}$ Richter et al derived site-specific and patient-specific CBCT calibration curves by comparing pixel values in patient CBCT images with corresponding regions of interest (ROI) in their pCT images. ${ }^{8}$ An in-depth comparison between the dose calculation accuracy of standard and site-specific CBCT calibration curves was conducted by Mariska et al based on CBCT images generated by two different vendors for lung cancer patients. ${ }^{17}$ More intensive techniques have attempted to overcome the CBCT image quality issues by employing density override techniques. ${ }^{11,16,18}$ Many techniques are based on exploiting the accurate HU information contained within pCT images to correct the CBCT images. Marchant et al developed a software to correct CBCT images by comparing corresponding pCT and CBCT axial slices. ${ }^{15}$ One of the most elaborated methods is the use of deformable registration algorithms to deform the accurate HU information contained within the patient's pCT images to the anatomical configuration recorded in the CBCT images. ${ }^{19,20}$ Deformable registration has the additional possibility to deform or "warp" the dose calculated on the CBCT anatomy back to a common reference anatomy (e.g. the $\mathrm{pCT}$ ) to determine an estimate of the dose accumulated over multiple treatment fractions. ${ }^{21,22}$

In this paper, we report on a comparative study of four commonly used techniques: standard pCT calibration curve, site-specific CBCT calibration curve, HU override and deformable registration. Each technique was evaluated in terms of accuracy for calculating the dose delivered to the target whilst minimizing the dose delivered to the organ at risk (OAR), for three treatment sites: head and neck, lung and prostate.

\section{METHODS AND MATERIALS}

Table 1 summarises the four techniques for performing dose calculations on CBCT images investigated in the present study. Each technique was applied to a sample of 15 patients who had received volumetric modulated arc therapy (VMAT) to one of three treatment sites (five head and neck, five lung and five prostate patients). Each patient had a pCT scan acquired using an Optima CT580 W CT scanner (GE Healthcare, Waukesha, WI, United States), with a four-dimensional CT acquisition protocol used to acquire pCT images for all lung patients. ${ }^{27}$ The pCT images were imported into Varian Eclipse (v13.5) (Varian Medical Systems, Palo Alto) for contouring and planning.

Four head and neck patients were prescribed 70 and 56 Gy in 35 fractions to their primary and nodal target volumes respectively, while one patient was prescribed 60 Gy in 30 fractions to a primary target volume only. All prostate patients were prescribed 74 and 60 Gy in 37 fractions to their prostate and pelvic lymph node target volumes, respectively. All lung patients were prescribed $55 \mathrm{~Gy}$ in 20 fractions to their target volume: the gross tumour volumes (GTVs) were outlined using the maximum intensity projections (MIPs) and phases, while OARs outlining and dose calculations were performed on the average CT images. ${ }^{27}$ Patients were treated using a TrueBeam v. 1.5 linear accelerator (Varian Medical Systems). Prior to treatment, CBCT images were acquired for the first three fractions and then weekly. The CBCT images were successively registered to the corresponding $\mathrm{pCT}$ images for setup error correction.

Throughout this investigation, the dose distributions calculated on the patient's pCT were considered as the gold-standard for dosimetric comparison. Therefore, the image difference tool in Eclipse was used to compare pCT and CBCT images and identify 15 patients (five for each treatment site) with CBCTs displaying anatomical configurations that closely matched their pCT. Consideration was given to both the patient's skin surface outline and internal anatomy structure to reduce the impact of anatomical changes on the dose calculations. All dose calculations were performed using the type-B analytical anisotropic algorithm (AAA). Due to the limited transverse field of view (FOV) of the CBCT images, the images were extended by $3 \mathrm{~cm}$ in the superior-inferior direction to reduce the effect of $\mathrm{CBCT}$ anatomical cropping. As this extension region did not include any anatomical data, the patient's body contour from

Table 1. Summary of the methods employed in this study to calculate treatment doses on CBCT anatomy

\begin{tabular}{|l|l|l|c|}
\hline Method & \multicolumn{1}{|c|}{ Description } & \multicolumn{1}{|c|}{ Additional requirements } & \multicolumn{1}{c|}{ References } \\
\hline Standard pCT calibration curve & Dose calculated using standard pCT calibration curve & None & $8,9,11,23$ \\
\hline $\begin{array}{l}\text { CBCT site-specific calibration } \\
\text { curve }\end{array}$ & $\begin{array}{l}\text { Dose calculated using calibration curve specifically } \\
\text { derived for CBCT images }\end{array}$ & $\begin{array}{l}\text { Calibration phantom/registered } \\
\text { pCT-CBCT patient images }\end{array}$ & $7,11,16,17$ \\
\hline HU override & $\begin{array}{l}\text { CBCT pixel data overridden using regions of interest or } \\
\text { alternative bulk method. }\end{array}$ & None & $13,17,18,24,25$ \\
\hline Deformable registration & pCT image deformed to CBCT anatomy. & Deformable registration software & $8,23,26$ \\
\hline
\end{tabular}

CBCT, cone beam CT; HU, Hounsfield unit. 
corresponding slices on their $\mathrm{pCT}$ was transferred to this region and a water $(\mathrm{HU}=0)$ density-override was applied to this structure.

\section{Standard PCT calibration curve}

The first technique employed the standard pCT HU to $\rho_{\mathrm{e}}$ calibration curve, i.e. a bilinear curve defined by three default Eclipse coordinates: $(-1000 \mathrm{HU}, 0),(100 \mathrm{HU}, 1.1)$ and $(6000 \mathrm{HU}, 3.92)$, routinely validated on the Optima CT scanner using the CIRS model 062M phantom (CIRS Inc., Norfolk, VA).

\section{Site-specific CBCT calibration curve}

For the second technique, the site-specific calibration curves were determined using a custom-made phantom, containing tissue-equivalent inserts of known electron density. The custommade phantom consisted of the CIRS model $062 \mathrm{M}$ phantom with wax filling the air gap between the cylindrical inserts protruding from the phantom surface (Figure A.1, A.2 in Supplementary Material 1). The phantom was placed between two $5 \mathrm{~cm}$ thick water-equivalent squared slabs following a procedure previously described by Rong et al. ${ }^{23}$ The average HU values of the inserts were measured for a selection of scatter conditions and $\mathrm{CBCT}$ acquisition protocols. Further details on the derivation of the site-specific calibration curves used in this investigation are described in Appendix A (Supplementary Material 1).

\section{HU override}

A density override approach was also applied in this investigation, where automatic contouring tools were used to delineate seven tissues/materials (air, lung, adipose, muscle, soft and dense bone and metal) on the CBCT images. The HU override values for these structures were determined from corresponding volumes automatically contoured on the patient's pCT (Appendix B, Supplementary Material 1).

\section{Deformable registration}

Finally, the Eclipse SmartAdapt workspace was used to deformably register the patient's pCT to the selected CBCT. Eclipse SmartAdapt consists of a toolset enabling to track and adapt changes in anatomy of a patient. It allows one to deform and propagate the pCT contours to match the current $\mathrm{CBCT}$ anatomy.

Figure 1 provides examples of pCT (1.a) and CBCT (1.b) slices together with $\mathrm{HU}$ overridden CBCT (1.c) and deformed pCT images (1.d) for a prostate (transversal view), head and neck (sagittal view) and lung (transversal view) cancer patient. For each technique, a 3 degrees of freedom rigid registration was used to register the $\mathrm{pCT}$ to the $\mathrm{CBCT}$ and transfer the original pCT structure set and treatment plan to the CBCT image sets. Dose distributions were then calculated for the original plan on the CBCT images using the AAA dose calculation algorithm.

Dose volume histogram (DVH) analysis was employed to compare the results of the dose calculations. DVHs for target and OAR structures were examined to quantify key plan characteristics. These characteristics were compared to values obtained from DVHs calculated using the original pCT data. In addition to this analysis, the plans and 3D dose distributions were exported and analysed using Verisoft v6.2 acquisition and analysis software (PTW, Freiburg, Germany). 3D $\gamma$ analysis was employed to compare the dose distributions calculated using each of the four techniques to the dose distributions calculated on the original pCT. A thresholding technique was used to subtract the failed pixels at the skin surface of the patient and at the edge of the imager. Following this, a $2.0 \% / 0.1 \mathrm{~mm} \gamma$ criterion was applied. Multiple dose thresholds (10\%, 50\%, 70 and 90\%) were used to evaluate the pCT-CBCT differences at different distances from the skin surface. Due to the limited FOV of the CBCT images, the volume of interest over which the $\gamma$ analysis was performed was limited to a length of $9 \mathrm{~cm}$ in the superoinferior direction to exclude the effect of $\mathrm{CBCT}$ anatomical cropping.

\section{RESULTS}

Figure 2 displays examples of DVHs acquired for representative patients who received radiotherapy to the prostate, head and neck, and lung. The CBCT DVHs for all the techniques overlap well with the pCT DVHs for all the OAR structures, however, differences are observed for the target structures. To provide a more quantitative comparison between the dose calculation techniques, selected DVH characteristics used to assess VMAT plan quality were extracted for all the target and OAR structures. Tables 2-4 report the median and ranges of differences between the original pCT and the CBCT DVHs calculated using each of the four techniques for key prostate and pelvic node, head and neck and lung plan quality criteria, respectively.

Prostate and pelvic node median differences for all target DVH metrics across all four techniques were within $0.6 \%$ (Table 2). Calculations performed using the standard and CBCT site-specific calibration curve were found to deviate from the $\mathrm{pCT}$ results by up to $+1.8 \%$ and $-1.4 \%$, respectively.

According to Table 3, for head and neck patients, the deformable registration technique provides the best agreement. Smaller median differences are observed for the primary target volume (DVH metrics within $0.4 \%$ ) while for the nodal target volume differences up to $5.4 \%$ from the pCT are calculated. Standard and site-specific CBCT calibration curve techniques are found to yield the poorest agreement. Larger differences (5.4\%, 3.9\%, 3.2 and $2.7 \%$ for standard curve, site-specific CBCT calibration curve, density override and deformable registration techniques, respectively) were observed for the dose delivered to $99 \%$ of the nodal target volumes.

In Table 4, absolute differences between pCT and CBCT DVHs for the lung patients are reported. The deformable registration and site-specific CBCT calibration techniques yield overall smaller median differences for the target volume and OARs, respectively. The results are consistently below $1.9 \%$, the only outlier being the maximum dose to $0.1 \mathrm{cc}$ of volume for the site-specific CBCT curve technique (3.8\%). No discernible differences are observed in the accuracy of the different techniques for the OAR's metrics.

Figure 3 displays a summary of the $\gamma$ analysis $2.0 \% / 0.1 \mathrm{~mm}$ results comparing the dose distributions calculated on the CBCT anatomy to distributions calculated on the original pCT images. 
Figure 1. (a) PCT slice of a prostate, head and neck and lung cancer patient. (b) Corresponding CBCT slice acquired using pelvis, head, and thorax acquisition protocol. (c) CBCT slice with HU overrides applied to the pixel data; specifically adipose and air HU overrides are here presented in orange and violet, respectively. (d) PCT data deformed to the CBCT anatomy with the deformation grid overlaid on the image. The PTV is contoured in red and blue (for prostate PTV74 is red and PTV60 is blue, for head and neck PTV70 is red and PTV56 is in blue), while the organs at risk are contoured in yellow (bladder, spinal cord, oesophagus), blue (brainstem, heart) and green (spinal cord). The couch is the purple element in each image. CBCT, cone beam CT; HU, Hounsfield unit; PTV, planning target volume.
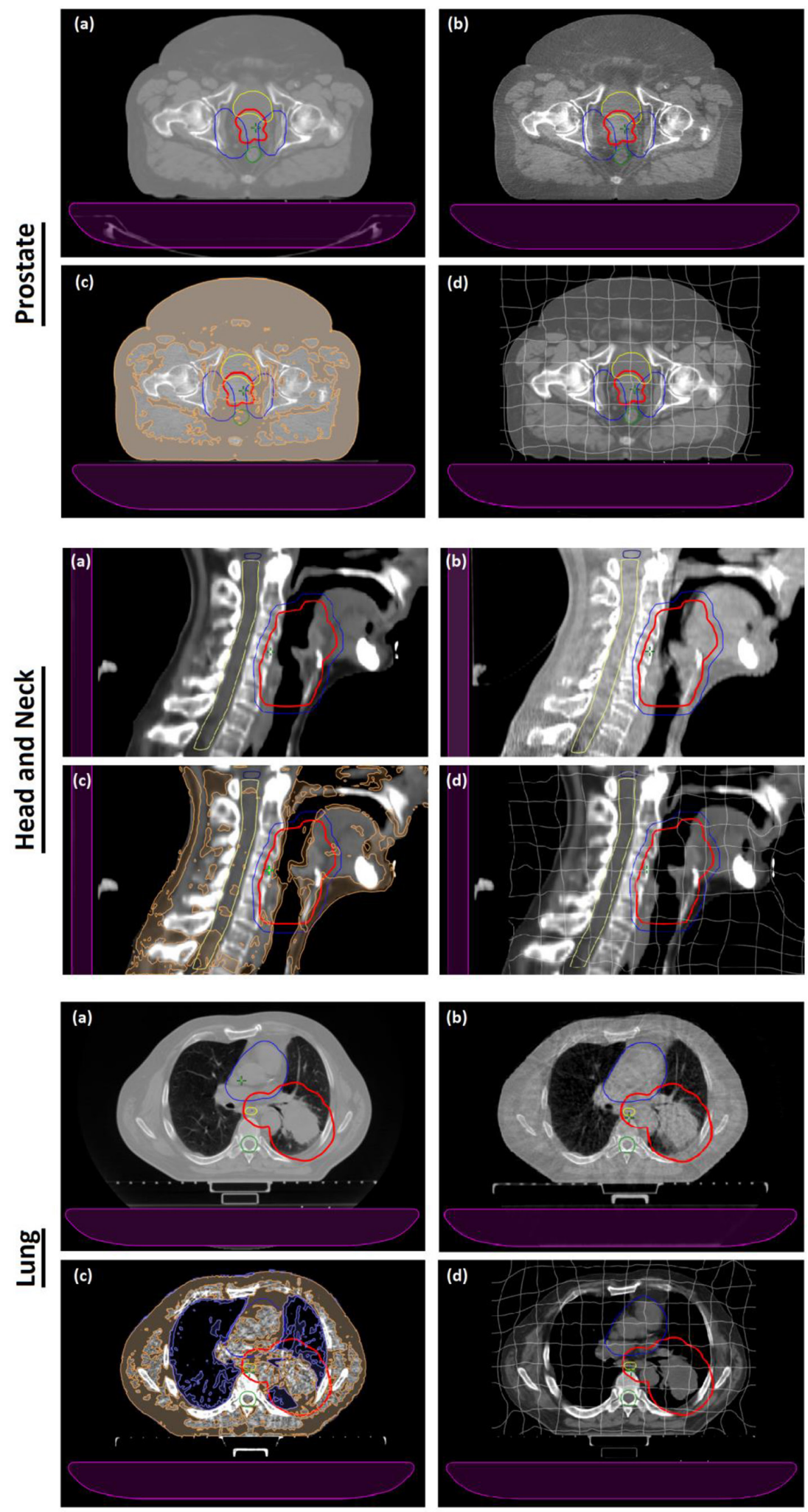
Figure 2. DVHs target volumes (PTV and nodal target volume) and OAR structures for (a,b) pelvis, (c,d) head, and (e,f) thorax acquisitions. OAR, organs at risk; DVH, dose volume histogram; PTV, planning target volume.

\section{$\underline{\text { Target Volumes }}$}

\section{Organs at Risk}

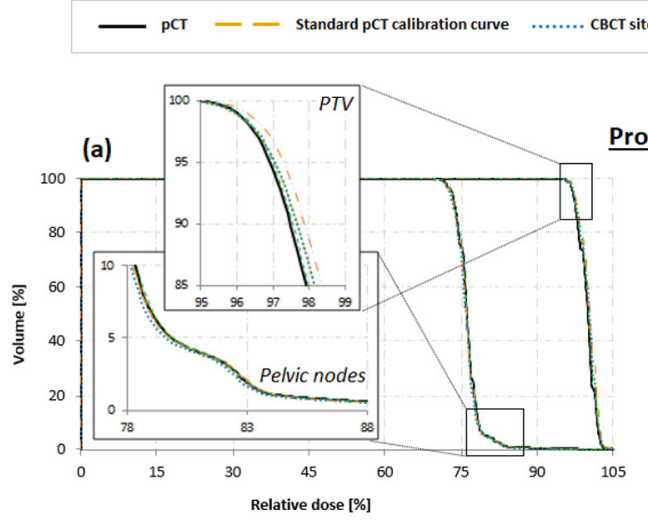

Prostate
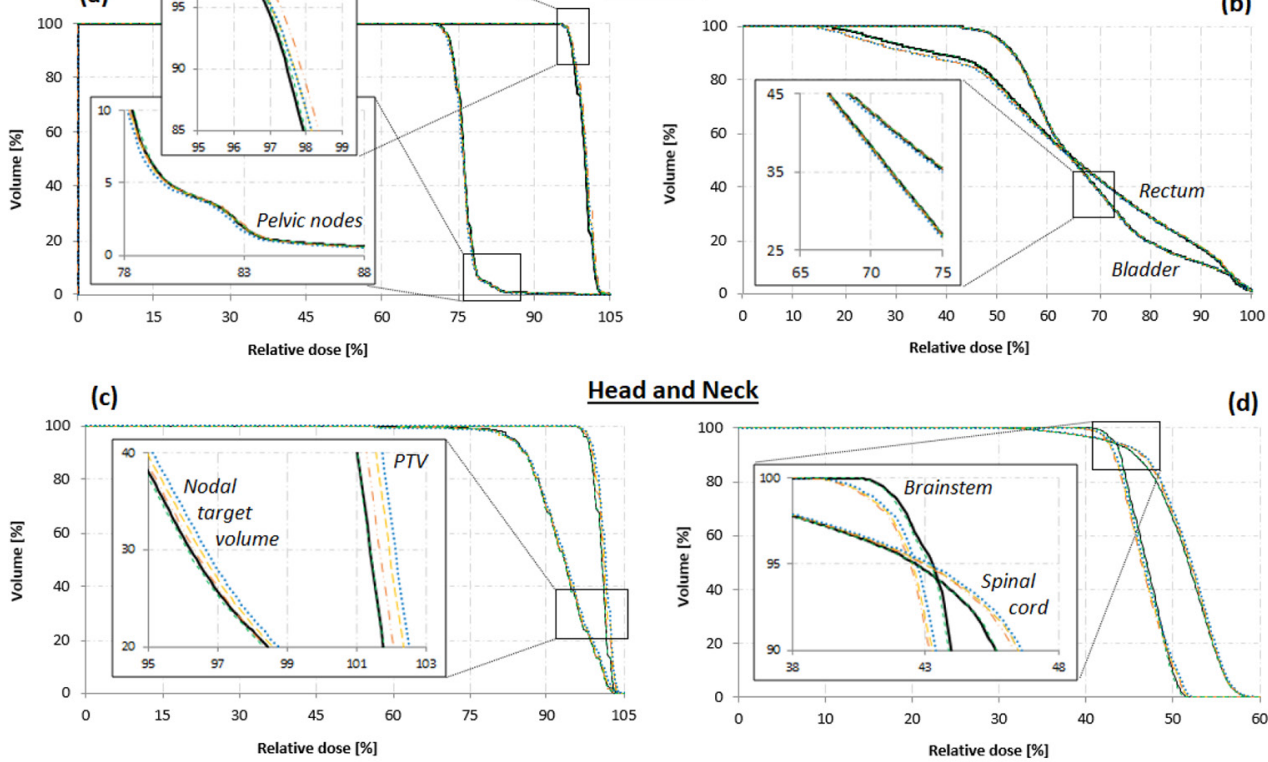

lead and Neck

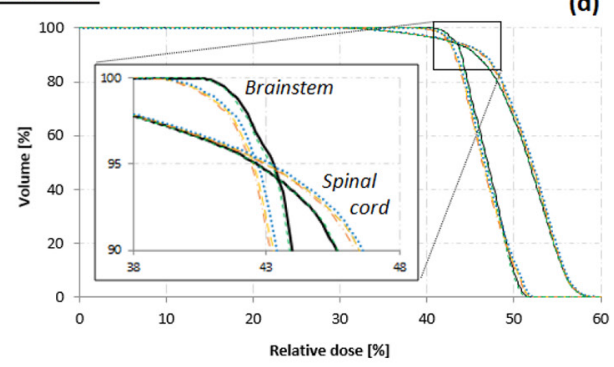

(e)

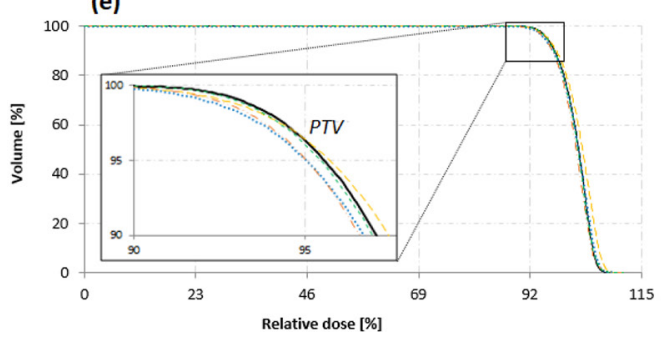

The median percentage pass-rates are reported for each of the three treatment sites, with minimum dose thresholds of $10 \%$, $50 \%, 70 \%$ or $90 \%$ applied to the $\gamma$ analysis. All four methods produce dose distributions that are a close match to the dose calculated using the pCT image, with pass-rates higher than $80 \%$ for all the chosen thresholds. For head and neck patients, the deformable registration technique gives the best results at any thresholds. For lung patients, the deformable registration technique gives the best results at 70 and $90 \%$ thresholds while at 10 and $50 \%$ the closest match to pCT are obtained using site-specific and standard calibration curves, respectively. Finally, for the prostate patients no discernible differences are observed. Moreover, it can be noted that $\gamma$ analysis performed on the standard calibration curve or the HU override dose distributions yield more variable pass-rates compared to the deformable registration. The pass-rate values displayed in Figure 3 and reported for each individual patient in Appendix C (Supplementary Material 1) also indicate a variation in the accuracy of each of the dose calculation techniques depending on the treated region.

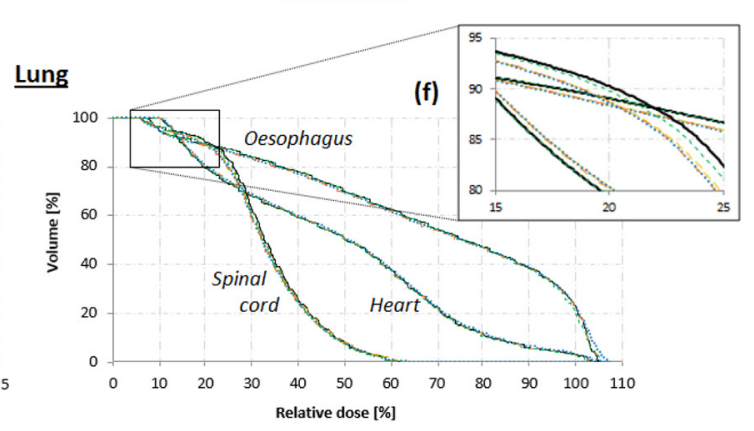

The median pass-rate observed at 50\% threshold are between 99.6-99.9\%, 94.6-96.0\%, and 94.8.0-96.0\% for prostate, head and neck and lung patients, respectively.

\section{DISCUSSION}

Radiotherapy treatment plans are typically created using CT images acquired on a pCT scanner. CBCT scans are taken pre-treatment to verify patient positioning although this volumetric image is not typically used to recalculate the dose due to the inferior image quality. The aim of this work was to determine the optimum method for performing treatment planning dose calculations using anatomical information contained within CBCT images.

Four different techniques were identified from the literature and evaluated to determine the best protocol to follow. With regards to efficiency, each technique required some precursor steps before dose calculations could be performed on the CBCT anatomy. Using the standard pCT, calibration curve was found 


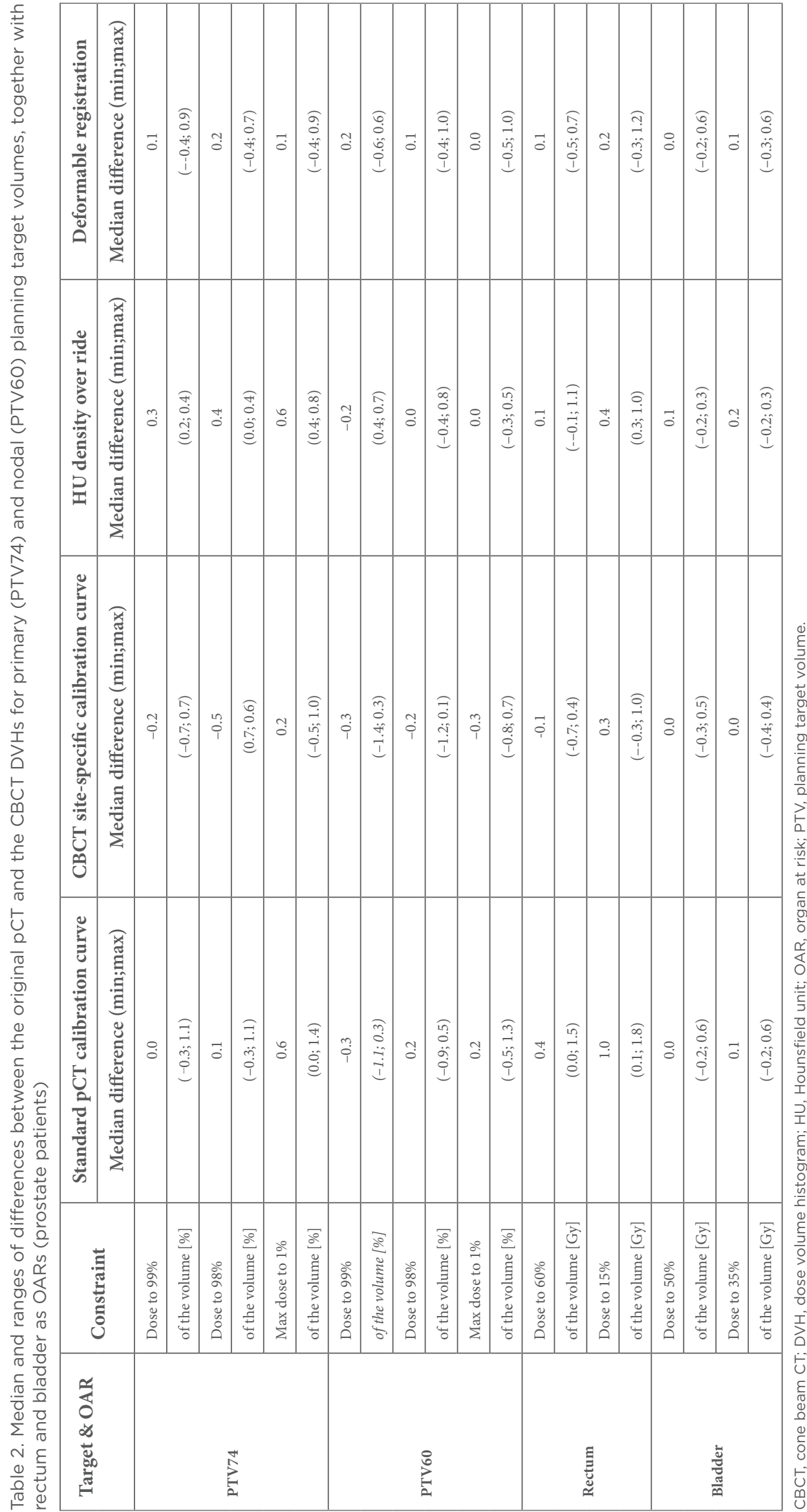


Table 3. Median and ranges of differences between the original PCT and the CBCT DVHs for primary (PTV70) and nodal (PTV56) planning target volumes, together with spinal cord and brain stem as OARs (head and neck patients)

\begin{tabular}{|c|c|c|c|c|c|}
\hline \multirow{2}{*}{$\begin{array}{l}\text { Target } \\
\text { \& OAR }\end{array}$} & \multirow{2}{*}{ Constraint } & $\begin{array}{l}\text { Standard pCT } \\
\text { calibration curve }\end{array}$ & $\begin{array}{l}\text { CBCT site-specific } \\
\text { calibration curve }\end{array}$ & $\begin{array}{l}\text { HU density } \\
\text { override }\end{array}$ & $\begin{array}{l}\text { Deformable } \\
\text { registration }\end{array}$ \\
\hline & & $\begin{array}{l}\text { Median difference } \\
\quad(\min ; \max )\end{array}$ & $\begin{array}{l}\text { Median difference } \\
\quad(\min ; \max )\end{array}$ & $\begin{array}{l}\text { Median difference } \\
\quad(\min ; \max )\end{array}$ & $\begin{array}{c}\text { Median } \\
\text { difference }\end{array}$ \\
\hline \multirow{6}{*}{ PTV70 } & Dose to $99 \%$ & -0.4 & -0.1 & 0.1 & 0.0 \\
\hline & of the volume [\%] & $(-0.8 ; 0.3)$ & $(-0.2 ; 0.5)$ & $(0.0 ; 0.3)$ & $(-0.2 ; 0.2)$ \\
\hline & Dose to $95 \%$ & -0.4 & 0.0 & 0.1 & 0.0 \\
\hline & of the volume [\%] & $(-0.6 ; 0.4)$ & $(-0.2 ; 0.7)$ & $(0.0 ; 0.3)$ & $(0.0 ; 0.4)$ \\
\hline & Max dose to $1 \%$ & 0.1 & 0.7 & 0.4 & 0.1 \\
\hline & of the volume [\%] & $(-0.1 ; 1.0)$ & $(0.2 ; 1.3)$ & $(0.3 ; 1.0)$ & $(0.0 ; 0.9)$ \\
\hline \multirow{6}{*}{ PTV56 } & Dose to $99 \%$ & -1.3 & -1.5 & -1.2 & -2.0 \\
\hline & of the volume [\%] & $(-5.4 ;-0.9)$ & $(-3.9 ;-0.3)$ & $(-3.2 ;-0.1)$ & $(-2.7 ;-0.2)$ \\
\hline & Dose to $95 \%$ & -0.2 & 0.0 & -0.2 & -0.4 \\
\hline & of the volume [\%] & $(-1.1 ; 0.0)$ & $(0.5 ; 0.3)$ & $(-0.3 ; 0.3)$ & $(-0.5 ;-0.1)$ \\
\hline & Max dose to $1 \%$ & 0.2 & 0.5 & 0.5 & 0.2 \\
\hline & of the volume [\%] & $(-0.2 ; 0.7)$ & $(0.1 ; 1.3)$ & $(0.3 ; 0.7)$ & $(0.0 ; 0.7)$ \\
\hline \multirow{2}{*}{ Spinal cord } & Dose to $0.1 \mathrm{~cm}^{3}$ & -0.2 & 0.0 & 0.0 & 0.0 \\
\hline & of volume [Gy] & $(-0.4 ; 0.1)$ & $(-0.1 ; 0.2)$ & $(-0.1 ; 0.1)$ & $(0.0 ; 0.1)$ \\
\hline \multirow{2}{*}{ Brain stem } & Dose to $0.1 \mathrm{~cm}^{3}$ & -0.1 & 0.2 & 0.2 & -0.1 \\
\hline & of the volume [Gy] & $(-0.2 ; 0.4)$ & $(0.1 ; 0.5)$ & $(0.0 ; 0.3)$ & $(-0.3 ; 0.1)$ \\
\hline
\end{tabular}

CBCT, cone beam CT; DVH, dose volume histogram; HU, Hounsfield unit; OAR, organ at risk; PTV, planning target volume.

Table 4. Median and ranges of differences between the original $\mathrm{PCT}$ and the CBCT DVHs for primary planning target volume (PTV55), together with spinal cord, oesophagus and heart as OARs (lung patients)

\begin{tabular}{|c|c|c|c|c|c|}
\hline \multirow{2}{*}{$\begin{array}{l}\text { Target } \\
\text { \& OAR }\end{array}$} & \multirow{2}{*}{ Constraint } & $\begin{array}{l}\text { Standard pCT } \\
\text { calibration curve }\end{array}$ & $\begin{array}{l}\text { CBCT site-specific } \\
\text { calibration curve }\end{array}$ & $\begin{array}{l}\text { HU density } \\
\text { override }\end{array}$ & $\begin{array}{l}\text { Deformable } \\
\text { registration }\end{array}$ \\
\hline & & $\begin{array}{l}\text { Median difference } \\
(\min ; \max )\end{array}$ & $\begin{array}{l}\text { Median difference } \\
\text { (min; max })\end{array}$ & $\begin{array}{l}\text { Median difference } \\
(\min ; \max )\end{array}$ & Median difference \\
\hline \multirow{6}{*}{ PTV55 } & Dose to $99 \%$ & -0.6 & -0.7 & -0.6 & -0.3 \\
\hline & of the volume [\%] & $(-1.0 ; 0.2)$ & $(-1.2 ;-0.4)$ & $(-1.1 ; 0.1)$ & $(-1.8 ; 0.9)$ \\
\hline & Dose to $95 \%$ & -0.4 & -0.5 & -0.5 & 0.1 \\
\hline & of the volume [\%] & $(-0.5 ;-0.1)$ & $(-0.8 ;-0.2)$ & $(-0.9 ; 0.4)$ & $(-0.3 ; 1.2)$ \\
\hline & Max dose to $0.01 \mathrm{~cm}^{3}$ & 0.7 & 2.3 & 0.4 & 0.9 \\
\hline & of the volume [\%] & $(0.5 ; 1.4)$ & $(1.4 ; 3.8)$ & $(-0.4 ; 1.2)$ & $(-0.8 ; 1.9)$ \\
\hline \multirow{2}{*}{ Spinal Cord } & Max dose to $0.01 \mathrm{~cm}^{3}$ & 0.2 & 0.0 & 0.0 & -0.2 \\
\hline & of volume [Gy] & $(-0.3 ; 0.7)$ & $(-0.6 ; 0.3)$ & $(-1.0 ; 0.4)$ & $(-0.7 ; 0.1)$ \\
\hline \multirow{2}{*}{ Oesophagus } & Max dose to $0.1 \mathrm{~cm}^{3}$ & 0.1 & 0.0 & 0.0 & 0.1 \\
\hline & of the volume [Gy] & $(-0.1 ; 1.9)$ & $(-0.2 ; 1.2)$ & $(-0.4 ; 1.5)$ & $(-0.3 ; 0.4)$ \\
\hline \multirow{2}{*}{ Heart } & Max dose to $0.1 \mathrm{~cm}^{3}$ & 0.5 & 0.0 & 0.1 & -0.1 \\
\hline & of the volume [Gy] & $(-0.1 ; 1.5)$ & $(-0.2 ; 1.3)$ & $(-0.3 ; 1.3)$ & $(-0.5 ; 0.5)$ \\
\hline
\end{tabular}

$\mathrm{CBCT}$, cone beam CT; DVH, dose volume histogram; HU, Hounsfield unit; OAR, organ at risk; PTV, planning target volume. 
Figure 3. Median $\gamma 2 \% / 0.1 \mathrm{~mm}$ pass-rates (\%) for dose thresholds of 10\%, 50\%, 70 and $90 \%$ for (a) pelvis (b) head and neck, and (c) thorax acquisitions. Note that the median pass-rate ( $y$ axis) starts from 60\%. CBCT, cone beam CT.
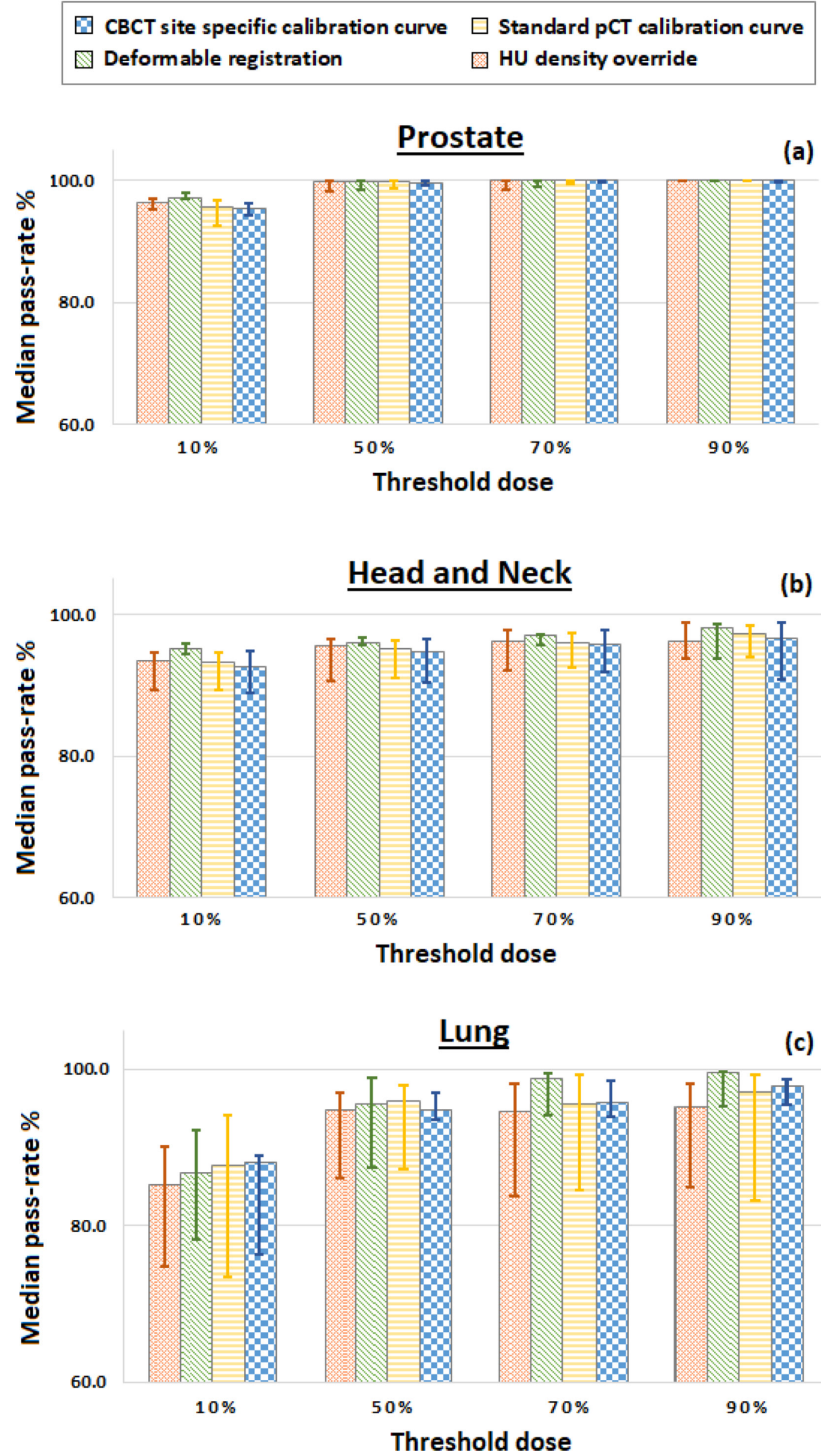
to be the most straightforward method to implement. However, for some acquisition protocols it was necessary to extend the CBCTs limited FOV to allow transfer of pCT structures with density overrides applied for improved calculation accuracy. ${ }^{16}$ This additional step was also required for the site-specific calibration curve and density override techniques but was inherent in the deformable registration technique. Deriving appropriate $\mathrm{CBCT}$ calibration curves required an additional investigation using a custom-made CBCT phantom. In agreement with previous work, ${ }^{23}$ site-specific variations in the calibration curves were observed and suggested the need for protocol specific curves. The density override method was found to be the most labour-intensive technique to employ for this system, although other manufacturers automate this step. ${ }^{16}$ A patient specific override approach was adopted as this had previously been reported to be more accurate than a population approach. ${ }^{8}$ Appendix B (Supplementary Material 1) provides more detail on the steps involved when employing this method. However, this process could potentially be streamlined through the use of scripts within the treatment planning system. ${ }^{28}$ Deformable registration required additional software to deform $\mathrm{pCT}$ images to the CBCT anatomy and generate new DICOM images which were subsequently imported into the treatment planning system. However, open source software for deformable registration is available and commercial systems offer the capability of automatically performing deformation and import. ${ }^{29}$

Quantitative DVH comparisons and $\gamma$ analysis were employed to assess the accuracy of each of the four techniques. The use of deformable registration consistently generated dose distributions that exhibited a good agreement with the pCT dose distributions (less than $0.3 \%$ of difference on the dose to $99 \%$ of volume, and average $\gamma$ pass-rate $>95 \%$ for all tumour sites). For most of the patients using either the standard calibration curve or applying density overrides to the CBCT images yielded very similar differences when compared to the $\mathrm{pCT}$ results. The use of a standard pCT calibration curve could be used where there is poor registration accuracy and density override could be a good alternative where CBCT images present significant artefacts, e.g. in case of prosthetic implants. Both the DVH metrics and $\gamma$ analysis indicated that applying a density override to the CBCT pixel data improved the calculation accuracy, highlighting the CT number accuracy issues associated with CBCT. ${ }^{4,9}$ The CBCT site-specific calibration curves derived using a custom-made phantom were observed to produce wider DVH metrics ranges but good $\gamma$ analysis results. It must be taken into account that when generating the site-specific calibration curves used for the dose recalculation, some ring and strike artefacts in the CBCT images caused worse $\mathrm{HU}$ measurements for some inserts. This can explain why the site-specific DVH results are broader than the other methods. The custom-made phantom used to generate the site-specific curves allowed improving the results presented by Rong et $\mathrm{al}^{23}$ because the additional scatter material resembled a real case scenario and the dose was recalculated on patients' images and not on phantoms. DVH analysis of the dose to $99 \%$ of volume of the nodal PTV volume in head and neck treatment plans presented the largest discrepancies, irrespective of the technique used, with dose differences up to $5.4 \%$ for the patients.
The source of this difference was found to be associated with the PTV position: the closer the PTV to the patient's skin surface, the larger the error. Hence, the dose calculated for this structure was more sensitive to small changes in the patient's CBCT geometry.

One potential limitation to this work is that the deformable registration study deformed the original pCT to the CBCT anatomy with the calculated deformed pCT dose subsequently compared to the dose calculated on the original pCT. This introduced an inherent bias as it was not possible to determine if agreement was partially due to the fact that the deformation algorithm did not sufficiently adjust the pCT anatomy. However, the accuracy of deformable registration techniques, including SmartAdapt ${ }^{\circledast}$, have been extensively tested, ${ }^{20,21,30,31}$ where a number of deformable registration algorithms have been reported to perform at an accuracy equivalent to the image voxel size. ${ }^{31}$ Arbitrary selection of CBCT images would have resulted in deviations from the $\mathrm{pCT}$ anatomy and influenced the dose distributions generated on the images. ${ }^{12}$ This work sought to eliminate the influence of anatomical changes on $\mathrm{CBCT}$ dose distributions and provide a more robust baseline assessment of dose calculation methods. Future work will assess multiple CBCT images from individual patients to assess the influence of anatomical changes on the dose distributions generated during individual treatment fractions.

It is also important to highlight that outlining on CBCT may not be feasible within the clinical workflow of a busy radiotherapy department. Therefore, automated knowledge-based contouring algorithms, based on deformable registration to appropriate reference cases, may provide a tool to streamline this process. ${ }^{32}$ Other studies have also observed vendor-specific variation in the quality of CBCT image acquisitions ${ }^{17,33}$ which may influence the accuracy of each of the dose calculation techniques assessed here. It is therefore recommended that radiotherapy departments perform similar investigations using their own CBCT image database to identify the best technique for their resources.

In vivo dosimetry is another important application of CBCT. Changes in patient geometry, dose calculation inaccuracies and dose delivery errors are all sources of uncertainty causing discrepancies between planned and delivered dose. ${ }^{34}$ Several studies have shown that the measurements with portal imaging device of the radiation dose received by the patient during the treatment allow the detection of errors that could remain undetected even using pre-treatment verification. ${ }^{35-37}$ The knowledge of in-room 3D imaging dose measurements and TPS 3D dose distributions (based on the actual delivered fields) can yield accurate 3D in-vivo patient dose verification, particularly useful for advanced photon beam techniques characterized by steeper dose gradients (e.g. IMRT and VMAT) ${ }^{38-40}$ The work presented in this manuscript shows that dose calculation on CBCT can introduce uncertainties, independent of delivery uncertainties or anatomical changes, that are dependent on the dose reconstruction technique.

\section{CONCLUSION}

In addition to providing superior 3D internal anatomy information for improved patient set-up, CBCT images can potentially 
be utilised to calculate more accurate estimates of the dose patients receive during their treatments. This work shows how to enable CBCT images to be used for this purpose with the potential for adapting treatments if required. The results of this study indicate that deformable registration is an accurate method for performing dose calculations on CBCT. HU override techniques and the use of site-specific CBCT calibration curves are valid alternatives to be considered with regards to accuracy and efficiency, particularly when substantial anatomical differences occur.

It is important to underline that the site of the tumour plays an important role in identifying the best technique to be used. Specifically (a) for prostate patients all four methods give very good and similar results; (b) for head and neck patients, the position of the tumour plays a relevant role in the dose recalculation. When delivering high doses close to the skin surface of the patient, geometrical differences between $\mathrm{pCT}$ and $\mathrm{CBCT}$ images can cause significant discrepancies in the dose recalculation; (c) for lung patients, the size of the patients can influence the CBCT dose recalculation, particularly in the recalculation of the dose delivered to the primary target.

\section{ACKNOWLEDGMENT}

Work was performed using treatment planning workstations purchased through charitable funds provided by Friends of the Cancer Centre (Registered with The Charity Commission for Northern Ireland NIC101345).

\section{FUNDING}

This work was supported by grants from Prostate Cancer UK and the Movember foundation (grant number: CEO13_2-004 (FASTMAN Centre)) and the R \& D division of the Public Health Agency (grant number: COM/4965/14).

\section{REFERENCES}

1. Jaffray DA, Drake DG, Moreau M, Martinez AA, Wong JW. A radiographic and tomographic imaging system integrated into a medical linear accelerator for localization of bone and soft-tissue targets. Int J Radiat Oncol Biol Phys 1999; 45: 773-89. doi: https://doi.org/10.1016/S0360-3016(99) 00118-2

2. Jaffray DA, Siewerdsen JH, Wong JW, Martinez AA. Flat-panel cone-beam computed tomography for imageguided radiation therapy. Int J Radiat Oncol Biol Phys 2002; 53(no. 5): 1337-49. doi: https://doi.org/10.1016/S0360-3016(02) 02884-5

3. Maund IF, Benson RJ, Fairfoul J, Cook J, Huddart R, Poynter A. Imageguided radiotherapy of the prostate using daily CBCT: the feasibility and likely benefit of implementing a margin reduction. Br J Radiol 2014; 87: 20140459. doi: https://doi.org/10.1259/bjr. 20140459

4. Srinivasan K, Mohammadi M, Shepherd J. "Cone beam computed tomography for adaptive radiotherapy treatment planning". J Med Biol Eng 2014; 34: 377-85. doi: https:// doi.org/10.5405/jmbe.1372

5. Yoo S, Yin FF. Dosimetric feasibility of cone-beam CT-based treatment planning compared to CT-based treatment planning. Int J Radiat Oncol Biol Phys 2006; 66: 155361. doi: https://doi.org/10.1016/j.ijrobp.2006. 08.031

6. Ding GX, Duggan DM, Coffey CW, Deeley M, Hallahan DE, Cmelak A, et al. A study on adaptive IMRT treatment planning using kV cone-beam CT. Radiother Oncol 2007; 85:
116-25. doi: https://doi.org/10.1016/j.radonc. 2007.06.015

7. van Zijtveld M, Dirkx M, Heijmen B. Correction of conebeam CT values using a planning CT for derivation of the "dose of the day". Radiother Oncol 2007; 85: 195-200. doi: https://doi.org/10.1016/j.radonc.2007.08. 010

8. Richter A, Hu Q, Steglich D, Baier K, Wilbert J, Guckenberger $M$, et al. Investigation of the usability of conebeam CT data sets for dose calculation. Radiat Oncol 2008; 3: 42-13. doi: https://doi.org/10.1186/1748-717X-3-42

9. Hatton J, McCurdy B, Greer PB. Cone beam computerized tomography: the effect of calibration of the Hounsfield unit number to electron density on dose calculation accuracy for adaptive radiation therapy. Phys Med Biol 2009; 54: N329-N346. doi: https://doi.org/ 10.1088/0031-9155/54/15/N01

10. Qian J, Lee L, Liu W, Chu K, Mok E, Luxton $\mathrm{G}$, et al. Dose reconstruction for volumetric modulated arc therapy (VMAT) using conebeam CT and dynamic log files. Phys Med Biol 2010; 55: 3597-610. doi: https://doi.org/ 10.1088/0031-9155/55/13/002

11. Fotina I, Hopfgartner J, Stock M, Steininger T, Lütgendorf-Caucig C, Georg D. Feasibility of CBCT-based dose calculation: comparative analysis of HU adjustment techniques. Radiother Oncol 2012; 104: 249-56. doi: https://doi.org/10.1016/j.radonc. 2012.06.007

12. Hatton JA, Greer PB, Tang C, Wright P, Capp A, Gupta S, et al. Does the planning dosevolume histogram represent treatment doses in image-guided prostate radiation therapy? Assessment with cone-beam computerised tomography scans. Radiother Oncol 2011; 98: 162-8. doi: https://doi.org/10.1016/j.radonc. 2011.01 .006

13. Hunter KU, Fernandes LL, Vineberg KA, McShan D, Antonuk AE, Cornwall C, et al. Parotid glands dose-effect relationships based on their actually delivered doses: implications for adaptive replanning in radiation therapy of head-and-neck cancer. Int J Radiat Oncol Biol Phys 2013; 87: 676-82. doi: https://doi.org/10.1016/j.ijrobp.2013.07. 040

14. Godley A, Ahunbay E, Peng C, Li XA. Automated registration of large deformations for adaptive radiation therapy of prostate cancer. Med Phys 2009; 36: 1433-41. doi: https://doi.org/10.1118/1.3095777

15. Marchant TE, Moore CJ, Rowbottom CG, MacKay RI, Williams PC. Shading correction algorithm for improvement of cone-beam CT images in radiotherapy. Phys Med Biol 2008; 53: 5719-33. doi: https://doi.org/10. 1088/0031-9155/53/20/010

16. Dunlop A, McQuaid D, Nill S, Murray J, Poludniowski G, Hansen VN, et al. Comparison of CT number calibration techniques for CBCT-based dose calculation. Strahlenther Onkol 2015; 191: 970-8. doi: https://doi.org/10.1007/s00066-015-0890-7

17. de Smet M, Schuring D, Nijsten S, Verhaegen F. Accuracy of dose calculations on $\mathrm{kV}$ cone beam CT images of lung cancer patients. Med Phys 2016; 43: 5934-41. doi: https://doi. org/10.1118/1.4964455

18. Chen S, Le Q, Mutaf Y, Lu W, Nichols EM, Yi BY, et al. Feasibility of CBCT-based dose with a patient-specific stepwise HU-to-density curve to determine time of 
replanning. J Appl Clin Med Phys 2017; 18: 64-9. doi: https://doi.org/10.1002/acm2. 12127

19. Kessler ML. Image registration and data fusion in radiation therapy. Br J Radiol 2006; 79(special_issue_1):S99-S108. doi: https:// doi.org/10.1259/bjr/70617164

20. Castadot P, Lee JA, Parraga A, Geets X, Macq B, Grégoire V. Comparison of 12 deformable registration strategies in adaptive radiation therapy for the treatment of head and neck tumors. Radiother Oncol 2008; 89: 1-12. doi: https://doi.org/10.1016/j.radonc.2008.04.010

21. Janssens G, de Xivry JO, Fekkes S, Dekker A, Macq B, Lambin P, et al. Evaluation of nonrigid registration models for interfraction dose accumulation in radiotherapy. Med Phys 2009; 36: 4268-76. doi: https://doi.org/10. $1118 / 1.3194750$

22. Velec M, Moseley JL, Craig T, Dawson LA, Brock KK. Accumulated dose in liver stereotactic body radiotherapy: positioning, breathing, and deformation effects. Int $J$ Radiat Oncol Biol Phys 2012; 83: 1132-40. doi: https://doi.org/10.1016/j.ijrobp.2011.09. 045

23. Rong Y, Smilowitz J, Tewatia D, Tomé WA, Paliwal B. Dose calculation on $\mathrm{kV}$ cone beam CT images: an investigation of the $\mathrm{Hu}$-density conversion stability and dose accuracy using the site-specific calibration. Med Dosim 2010; 35: 195-207. doi: https:// doi.org/10.1016/j.meddos.2009.06.001

24. Veiga C, McClelland J, Moinuddin S, Lourenço A, Ricketts K, Annkah J, et al. Toward adaptive radiotherapy for head and neck patients: Feasibility study on using CTto-CBCT deformable registration for "dose of the day" calculations. Med Phys 2014; 41: 031703. doi: https://doi.org/10.1118/1. 4864240

25. Almatani T, Hugtenburg RP, Lewis R, Barley S, Edwards M. Simplified material assignment for cone beam computed tomography-based dose calculations of prostate radiotherapy with hip prostheses.
J Radiother Pract 2016; 15: 170-80. doi: https://doi.org/10.1017/S1460396915000564

26. Onozato Y, Kadoya N, Fujita Y, Arai K, Dobashi S, Takeda K, et al. Evaluation of onboard $\mathrm{kV}$ cone beam computed tomographybased dose calculation with deformable image registration using Hounsfield unit modifications. Int J Radiat Oncol Biol Phys 2014; 89: 416-23. doi: https://doi.org/10. 1016/j.ijrobp.2014.02.007

27. Cole AJ, Hanna GG, Jain S, O'Sullivan JM. Motion management for radical radiotherapy in non-small cell lung cancer. Clin Oncol 2014; 26: 67-80. doi: https://doi.org/10.1016/ j.clon.2013.11.001

28. Almatani T, Hugtenburg RP, Lewis RD, Barley SE, Edwards MA. Automated algorithm for CBCT-based dose calculations of prostate radiotherapy with bilateral hip prostheses. Br J Radiol 2016; 89: 20160443. doi: https://doi.org/10.1259/bjr.20160443

29. Zukauskaite R, Brink C, Hansen CR, Bertelsen A, Johansen J, Grau C. "OpenSource-System für die deformierbare bildregistrierung von planungs- und rezidivCT-datensätzen: Validierung im kopf-halsbereich". Strahlentherapie und Onkol 2016; 192: 545-51.

30. Ramadaan IS, Peick K, Hamilton DA, Evans J, Iupati D, Nicholson A, et al. Validation of Varian's SmartAdapt ${ }^{\circledR}$ deformable image registration algorithm for clinical application. Radiat Oncol 2015; 10: 1-9. doi: https://doi.org/10.1186/s13014-015-0372-1

31. Brock KK, Deformable Registration Accuracy Consortium. Results of a multiinstitution deformable registration accuracy study (MIDRAS). Int J Radiat Oncol Biol Phys 2010; 76: 583-96. doi: https://doi.org/ 10.1016/j.ijrobp.2009.06.031

32. Haas B, Coradi T, Scholz M, Kunz P, Huber $\mathrm{M}$, Oppitz U, et al. Automatic segmentation of thoracic and pelvic CT images for radiotherapy planning using implicit anatomic knowledge and organ-specific segmentation strategies. Phys Med Biol 2008;
53: 1751-71. doi: https://doi.org/10.1088/ 0031-9155/53/6/017

33. Gardner SJ, Studenski MT, Giaddui T, Cui Y, Galvin J, Yu Y, et al. Investigation into image quality and dose for different patient geometries with multiple cone-beam CT systems. Med Phys 2014; 41: 31908. doi: https://doi.org/10.1118/1.4865788

34. van Elmpt W, Nijsten S, Petit S, Mijnheer B, Lambin P, Dekker A. 3D in vivo dosimetry using megavoltage cone-beam CT and EPID dosimetry. Int J Radiat Oncol Biol Phys 2009; 73: 1580-7. doi: https://doi.org/10.1016/j. ijrobp.2008.11.051

35. Mans A, Wendling M, McDermott LN, Sonke JJ, Tielenburg R, Vijlbrief R, et al. Catching errors with in vivo EPID dosimetry. Med Phys 2010; 37: 2638-44. doi: https://doi. org/10.1118/1.3397807

36. Olaciregui-Ruiz I, Rozendaal R, Mijnheer $B$, van Herk M, Mans A. Automatic in vivo portal dosimetry of all treatments. Phys Med Biol 2013; 58: 8253-64. doi: https://doi.org/ 10.1088/0031-9155/58/22/8253

37. Spreeuw H, Rozendaal R, Olaciregui-Ruiz I, González P, Mans A, Mijnheer B, et al. Online 3D EPID-based dose verification: Proof of concept. Med Phys 2016; 43: 396974. doi: https://doi.org/10.1118/1.4952729

38. Mijnheer B, Beddar S, Izewska J, Reft C. In vivo dosimetry in external beam radiotherapy. Med Phys 2013; 40: 070903. doi: https://doi.org/10.1118/1.4811216

39. Fidanzio A, Porcelli A, Azario L, Greco F, Cilla S, Grusio M, et al. Quasi real time in vivo dosimetry for VMAT. Med Phys 2014; 41: 062103. doi: https://doi.org/10.1118/1. 4875685

40. Van Uytven E, Van Beek T, McCowan PM, Chytyk-Praznik K, Greer PB, McCurdy BM. Validation of a method for in vivo 3D dose reconstruction for IMRT and VMAT treatments using on-treatment EPID images and a model-based forward-calculation algorithm. Med Phys 2015; 42: 6945-54. doi: https://doi.org/10.1118/1.4935199 